\title{
Effect of NP and foliar spray on growth and chemical compositions of some medicinal Apiaceae plants grow in arid regions in Egypt
}

\author{
Khalid A. Khalid \\ Medicinal and Aromatic Plants Department, National Research Centre, El Buhouth St., 12311, Dokki, Cairo, Egypt. \\ *Corresponding author: ahmed490@gmail.com
}

\begin{abstract}
Arid regions in Egypt are characterized by poor nutrients such as macro and microelements and unfavorable environmental conditions which negatively affect growth and productivity of medicinal and aromatic plants including anise (Pimpinella anisum L.), coriander (Coriandrum sativum L.) and sweet fennel (Foeniculum vulgare var. Dolce) plants. Thus, the main objective of the present investigation was to study the effect of different levels of NP fertilizers, trace elements and their interactions on the morphological and biochemical contents of these three plants under arid regions conditions. The effects of NP and trace elements on the growth (height, leaf number, branch number, umbel number, fresh weight, dry weight and fruit yield per plant) was measured and quantitative analysis of essential oils, fixed oil, total carbohydrates, soluble sugars and nutrient content of anise, coriander and sweet fennel were performed. The most effective rate was $\mathrm{N} 3 \mathrm{P} 3 \mathrm{x}$ trace elements interaction, resulting in a positive increase in vegetative growth characters. The highest values of vegetative growth characters were $53.4,45.9,10.3,33.5,36.8,11.8$ and 7.9 , respectively for anise; 83.0, 69.3, 9.8, 29.0, 34.0, 17.5 and 14.4, respectively for coriander; 89.8, 32.6, 7.8, 22.9, 257.8, 99.1 and 27.8, respectively for sweet fennel. As well as N3P3 x trace elements led to higher biochemical contents than the control. The increases were $0.9,0.3$ and $0.9 \%$ in essential oil; 5.4, 4.4 and $3.7 \%$ in fixed oil, $9,7.9$ and $8.2 \%$ in total carbohydrates; $2.4,2.8$ and $1.6 \%$ in soluble sugars; $5.0,7.5$ and $14.4 \%$ in crude protein; $0.8,2.0$ and $2.3 \%$ in nitrogen; $1.5,0.6$ and $0.4 \%$ in phosphorous; $1.3,1.2$ and $1.7 \%$ in potassium for anise, coriander and sweet fennel, respectively.
\end{abstract}

Keywords: NP, trace elements, growth, essential oil, fixed oil, nutrients, crude protein, soluble sugars, total carbohydrates. 


\section{Introduction}

Plant nutrition one of the most important factors that increase plant production. Nitrogen $(\mathrm{N})$ is the most recognized in plant for its presence in the structure of the protein molecule. Accordingly, $\mathrm{N}$ plays an important role in synthesis of the plant constituents through the action of different enzymes (Jones et al., 1991). N fertilization has been reported to reduce essential oil content in creeping juniper (Juniperus horizontalis) (Robert, 1986), although it has been reported to increase total essential oil yield in thyme (Thymus vulgaris L.) (Baranauskienne et al., 2003). Baranauskienne et al., (2003) found that $\mathrm{N}$ fertilizer increased herb yield, but essential oil content was not remarkable of thyme (Thymus vulgaris). Ashraf et al., (2006) showed that $\mathrm{N}$ fertilization had a significant increase in the oil content but did not affect on the nutrient content of black cumin (Nigella sativa L.) seeds. Akbarinia et al., (2007) indicated that increase $\mathrm{N}$ up to $60 \mathrm{~kg}$ $\mathrm{ha}^{-1}$ caused a significant increase in coriander (Coriandrum sativum L.) seed yield, but the highest essential oil content and fatty acids were obtained with $90 \mathrm{~kg}$ $\mathrm{N} \mathrm{ha}^{-1}$. Senthil Kumar et al., (2009) revealed that $\mathrm{N}$ at $93.75 \mathrm{~kg} \mathrm{ha}^{-1}$ gave the highest values of plant height, number of laterals, fresh and dry weight of shoot, dry matter production, fresh herb yield and essential oil yield of Davana (Artemisia pallens Wall.), while the maximum fresh and dry weight of root was obtained with $93.75 \mathrm{~kg} \mathrm{ha}^{-1}$ of nitrogen. The highest values of vegetative growth, oil yield and NPK content of dill (Anethum graveolens L). plants were recorded by the treatment of $100 \mathrm{~kg} \mathrm{~N} \mathrm{ha}^{-1}$ (Hellal et al., 2011).

Seeds have the highest concentration of $\mathrm{P}$ in a mature plant, and $\mathrm{P}$ is required in large quantities in young cells, such as shoots and root tips, where metabolism is high and cell division is rapid. $\mathrm{P}$ aids in root development, flower initiation, seed and fruit development $\mathrm{P}$ has been shown to reduce disease incidence in some plants and has been found to improve the quality of certain crops (Silva and Uchida, 2000). P is found to be abundant in fruits and seeds of cucumber plants (Papadopoulos, 1994). It is widely found that increasing $\mathrm{P}$ as a fertilizer will promote reproductive yields (Egle et al., 1999) and inflorescence production (Besmer and Koide, 1999), particularly when P is limiting in natural systems (Feller, 1995). Conversely, limitation of $\mathrm{P}$ supply has been shown to decrease the production of floral structures (Arnon and Hoagland, 1943; Shamsi and Whitehead, 1977; Ma et al., 2001). $\mathrm{P}$ concentrations were manipulated in order to maximize flower-head yield of Calendula officinalis L.; it was found that high $\mathrm{P}$ concentrations did not increase flower production, but instead produced significantly more leaf biomass (Stewart and Lovett-Doust, 2003). Three different concentrations of $\mathrm{P}(5,30$, and $60 \mathrm{mg}$ $\left.\mathrm{L}^{-1}\right)$ in the nutrient solution were used for the cultivation of Origanum dictamnus, significant differences (qualitative and quantitative) were observed between the essential oil samples (Economakis et al., 2002). The five levels of $\mathrm{P}$ evaluated were 0, 30, 60, 90 and $120 \mathrm{~kg} \mathrm{P}_{2} \mathrm{O}_{5}$ ha $^{-1}$ using single super phosphate fertilizer $(8 \% \mathrm{P})$, statistical analysis showed that $90 \mathrm{~kg} \mathrm{P}_{2} \mathrm{O}_{5}$ $\mathrm{ha}^{-1}$ gave statistically significant higher fruit yield of Trichosanthes cucumerina L. compared with other P levels (Adebooye and Oloyede, 2007). The Catharanthus roseus plants treated with 150 and $200 \mathrm{~kg}$ $\mathrm{P}_{2} \mathrm{O}_{5}$ ha $^{-1}$ had the maximum plant height, number of leaves, root biomass, $\mathrm{P}$ content when compared with the control plants (Karthikeyan et al., 2008). $150 \mathrm{~kg}$ $\mathrm{fed}^{-1}\left(\right.$ feddan $\left.=4200 \mathrm{~m}^{2}\right)$ of superphosphate calcium produced maximum fresh leaves yield of Artichoke (Cynara cardunculus var. scolymus) plants (Ezz ElDin et al., 2010).

Hornok (1980) indicated that NP fertilization not only effective on the quantity of vegetative and gen- 
eration mass, but on the essential oil content of dill (Anethum graveolens L.). The application of $100 \mathrm{~kg} \mathrm{~N}$ and $26 \mathrm{~kg} P$ per hectare produced the highest biomass and essential oil yields and NPK uptake of davana (Artemisia pallens Wall.) (Rao, 1989). The highest yields of inflorescence and essential oil of chamomile (Chamomilla recutita (L.) were achieved when the ratio between the major nutrients $\mathrm{N}$ : P was 1:1 (Nikolova et al., 1999). High amount of NP (2.0 and 4.0 $\left.\mathrm{g} \mathrm{pot}^{-1}\right)$ increased plant height, dry mass, and flower head yield of gum (Grindelia camporum Greene) plants (Mahmoud, 2002).

Micronutrients are involved in all metabolic and cellular functions. Plants differ in their need for micronutrients. Several of these elements are redox-active that makes them essential as catalytically active cofactors in enzymes, others have enzyme-activating functions, and yet others fulfill a structural role in stabilizing proteins (Hänsch and Mendel, 2009). Application of trace elements improved the performance of the plants; increased leaf size and yield of foxglove plant (Letchamo, 1986). Kandeel (1991) reported that using trace elements as foliar application at $2000 \mathrm{mg}$ $\mathrm{L}^{-1}+\mathrm{NP}$ had a significant effect on plant height, fresh weight, dry weight, fruit yield and essential oil content of parsley (Petroselinum crispum Mill).

Anise (Pimpinella anisum L., Apiaceae) has been used as an aromatic herb and spice since Egyptian times and antiquity and has been cultivated throughout Europe (Hänsel et al., 1999). In folk medicine, anise is used as an appetizer, tranquillizer and diuretic drug (Tyler et al., 1988; Lawless, 1999). For medical purposes, it used to treat dyspeptic complaints and catarrh of the respiratory tract, and as mild expectorants. It was also reported that extracts from anise fruits have therapeutic effects on several conditions, such as gynaecological and neurological disorders (Lawless, 1999; Czygan and Anis, 1992). Ethanolic extract of anise fruits con- tains trans-anethole, methylchavicol (estragole), eugenol, psedoisoeugenol, anisaldehyde, coumarins (umbelliferon, scopoletin), caffeic acid derivatives (chlorogenic acid), flavonoids, fatty oil, proteins, minerals, polyenes and polyacetylenes as its major compounds (Hänsel et al., 1999). Coriander (Coriandrum sativum $\mathrm{L}$.) is a culinary and medicinal plant belongs to the Apiacea family. This plant is of economic importance since it has been used as flavoring agent in food products, perfumes and cosmetics. As a medicinal plant, C. sativum L. has been credited with a long list of medicinal uses. Powdered seeds or dry extract, tea, tincture, decoction or infusion have been recommended for dyspeptic complaints, loss of appetite, convulsion, insomnia and anxiety (Emamghoreishi et al., 2005). Moreover, the essential oils and various extracts from coriander have been shown to possess antibacterial (Burt, 2004), antioxidant (Wangensteen et al., 2004), anticancerous and antimutagenic (Chithra and Leelamma, 2000) activities. Many phytochemical studies so far investigated the chemical composition of the essential oil from C. sativum L. fruits from different origins (Steinegger and Hansel, 1988). Evaluations of the essential oil composition extracted from leaves have also been reported (Eyres et al., 2005). The coriander (Coriandrum sativum L.) fruits essential oil yields showed marked increase during maturation process and linalool was the main compound at the fruiting stage (Kamel et al., 1994). Due to their unique and preferred favor and aroma, the swollen bases of sweet fennel (Foeniculum vulgare var. Dolce. Apiaceae) are freshly consumed in salads or cooked as a kitchen vegetable (Haupt, 1986; Marotti et al., 1993; Stuart, 1982). The major constituents of fennel essential oil such as anethole and limonene are also used as essence in cosmetics and perfumes and for some medicinal purposes (Marotti et al., 19 93; Stuart, 1982). 
Arid regions in Egypt are characterized by poor nutrients (macro and micro) and unfavorable environmental conditions which negatively affect growth and productivity of medicinal and aromatic plants including anise, coriander and sweet fennel plants (Abd-Allah et al., 2001). The main objective of the present investigation was to study the effect of different levels of NP fertilizers, trace elements and their interactions on the morphological and biochemical contents of anise, coriander and sweet fennel plants under these arid conditions.

\section{Materials and methods}

\subsection{Experimental}

Experiments were carried out in arid region at the Experimental Farm of Desert Development Center (DDC) in Sadat City, American University, Egypt, during two successive seasons, 1992/93 and 1993/94. The area of DDC had been recently reclaimed and had not cultivated before. Physical and chemical properties of the soil used in this study were determined according to Jackson (1973) and Cottenie et al., (1982) and are presented in Table 1.

Table 1. Mechanical and chemical analysis of the soil.

\begin{tabular}{|c|c|c|c|c|c|c|c|}
\hline \multicolumn{2}{|c|}{ Sand } & \multirow{2}{*}{$\begin{array}{c}\begin{array}{c}\text { Silt } \\
\%\end{array} \\
13.0 \\
\mathrm{Na}^{++}\end{array}$} & \multirow{2}{*}{$\begin{array}{c}\text { Clay } \\
7.3 \\
\mathbf{K}^{+} \\
\end{array}$} & \multirow{3}{*}{$\begin{array}{c}\text { Gravel } \\
18.7 \\
\mathrm{CO}_{3}\end{array}$} & \multirow{3}{*}{\begin{tabular}{|c|}
$\mathbf{p H}$ \\
8.7 \\
$\mathrm{HCO}_{3}^{-}$
\end{tabular}} & \multicolumn{2}{|c|}{$E C\left(d S m^{-1}\right)$} \\
\hline \multicolumn{2}{|c|}{79.7} & & & & & & \\
\hline \multicolumn{6}{|c|}{ meq $L^{-1}$} & & \\
\hline \multirow[t]{3}{*}{4.9} & 5.6 & 11.9 & 0.6 & 1.8 & 1.9 & 18.6 & 1.2 \\
\hline & & & $\mathrm{Fe}$ & $\mathrm{Cu}$ & $\begin{array}{c}\mathrm{Zn} \\
\mathrm{mg} \mathrm{L^{-1 }} \\
\end{array}$ & & \\
\hline & & & 5.4 & 0.4 & 0.3 & & \\
\hline
\end{tabular}

Seeds of coriander and anise, which were kindly provided by the Department of Medicinal and Aromatic Plants, Ministry of Agriculture, Giza, Egypt; whereas sweet fennel seeds were imported from France. Sweet fennel seeds were sown in the third week of October during both seasons. The seedlings of sweet fennel were transplanted into the open field 45 days after sowing. At the same time, the seeds of coriander and anise were sown directly in the open field. The experimental design was a complete randomized block with four replicates. The experimental area (plot) was 30 $\mathrm{m}^{2}(4 \mathrm{~m} \times 7.5 \mathrm{~m})$ containing 15 rows; the distance between hills was $25 \mathrm{~cm}$ and $50 \mathrm{~cm}$ apart. Thinning for two plants per hill was made 45 days after cultivating the plants in the open field. All agriculture practices operations other than experimental treatments were performed according to the recommendations of the Ministry of Agriculture, Egypt. Plots were divided into two main groups. The first group was subjected to different levels of NP combinations: N0P0, N1P1, N2P2 and N3P3. N0 $=0 \mathrm{~kg} \mathrm{~N} \mathrm{ha}^{-1}, \mathrm{~N} 1=100 \mathrm{~kg} \mathrm{~N} \mathrm{ha}^{-1}, \mathrm{~N} 2$ $=150 \mathrm{~kg} \mathrm{~N} \mathrm{ha}^{-1}, \mathrm{~N} 3=200 \mathrm{~kg} \mathrm{~N}^{-1} ; \mathrm{P} 0=0 \mathrm{~kg} \mathrm{P}_{2} \mathrm{O}_{5}$ ha $^{-1}, \mathrm{P} 1=37.5 \mathrm{~kg} \mathrm{P}_{2} \mathrm{O}_{5}$ ha $^{-1}, \mathrm{P} 2=56.3 \mathrm{~kg} \mathrm{P}_{2} \mathrm{O}_{5} \mathrm{ha}^{-1}, \mathrm{P} 3$ $=75 \mathrm{~kg} \mathrm{P}_{2} \mathrm{O}_{5} \mathrm{ha}^{-1}$. The second group was subjected to the same NP treatments but foliar spray (trace elements) was added at $1 \mathrm{~g} \mathrm{~L}^{-1}$. $\mathrm{N}$ source was ammonium sulphate $\left[\left(\mathrm{NH}_{4}\right)_{2} \mathrm{SO}_{4}\right](20 \% \mathrm{~N}) . \mathrm{P}_{2} \mathrm{O}_{5}$ source was calcium superphosphate $\left(15 \% \mathrm{P}_{2} \mathrm{O}_{5}\right)$. Foliar spray source 
was commercial solution (Greenzite) which contains EDTA Na2 Mn (40\%), EDTA Na2 Zn (48\%), Fe (5.4 $\left.\mathrm{mg} \mathrm{L} \mathrm{L}^{-1}\right), \mathrm{Mg}\left(0.54 \mathrm{mg} \mathrm{L}^{-1}\right), \mathrm{Mn}\left(50.54 \mathrm{mg} \mathrm{L}^{-1}\right), \mathrm{Zn}$ (570.27 $\left.\mathrm{mg} \mathrm{L}^{-1}\right), \mathrm{Cu}\left(0.054 \mathrm{mg} \mathrm{L}^{-1}\right)$, Mo (0.027 mg $\left.\mathrm{L}^{-1}\right)$, Ni (0.005 $\left.\mathrm{mg} \mathrm{L}^{-1}\right)$ and Co $\left(0.005 \mathrm{mg} \mathrm{L}^{-1}\right)$. Greenzite was added as foliar spray during 2 times, the first one after 2 weeks from thinning while the seconded one after 21 days from the first one.

\subsection{Harvesting}

At fruiting stage, the plants were harvested at the end of two seasons. Vegetative growth characters measurements [plant height $(\mathrm{cm})$, leaf number $\left(\right.$ plant $\left.^{-1}\right)$, branch number $\left(\right.$ plant $\left.^{-1}\right)$, umbel number (plant $\left.{ }^{1}\right)$, herb fresh weight $\left(\mathrm{g} \mathrm{plant}^{-1}\right)$, herb dry weight $\left(\mathrm{g} \mathrm{plant}^{-1}\right)$ and fruit yield $\left.\left(\mathrm{g} \mathrm{plant}^{-1}\right)\right]$ were recorded.

\subsection{Essential oil isolation}

Ripening fruits were collected from each treatment during the first and second season, and then $100 \mathrm{~g}$ from each replicate of all treatments was subjected to hydro-distillation for $3 \mathrm{~h}$ using a Clevenger type apparatus (Clevenger, 1928). The Essential oil content was calculated as a percentage.

\subsection{Total carbohydrates and soluble sugars}

Total carbohydrates and soluble sugars concentrations in leaves (collected at the end of the first and second season of each treatment) were determined according to Ciha and Brun (1978) with some modifications. Samples of $100 \mathrm{mg}$ were homogenized with $10 \mathrm{~mL}$ of extracting solution [glacial acetic acid: methanol: water, 1:4:5, v/v/v for TSS or glacial acetic acid: H2SO4 (1n): water, 1:4:5, v/v/v for TC]. The homogenate was centrifuged for $10 \mathrm{~min}$ at $3.000 \mathrm{rpm}$ and the supernatant was decanted. The residue was resuspended in
$10 \mathrm{~mL}$ of extracting solution and centrifuged another $5 \mathrm{~min}$ at $3.000 \mathrm{rpm}$. The supernatant was decanted, combined with the original extract and made up to 50 $\mathrm{mL}$ with water. For measurement of total carbohydrates and soluble sugars, a phenol-sulfuric acid assay was used (Dubois et al., 1956). A volume of $0.5 \mathrm{~mL}$ of $5 \%(\mathrm{v} / \mathrm{v})$ phenol solution and $2.5 \mathrm{~mL}$ of concentrated sulfuric acid were added to $0.5 \mathrm{~mL}$ aliquots. The mixture was shaken, heated in a boiling water-bath for 20 min and cooled to room temperature. The absorption was then determined by spectrophotometry at $490 \mathrm{~nm}$.

\subsection{Fixed oil, nutrients and protein determination}

Fixed oil extraction: $50 \mathrm{~g}$ of fruits were crushed to coarse powered and extracted with petroleum ether $\left(40-60{ }^{\circ} \mathrm{C}\right)$ in a Soxhlet apparatus (AOAC 1970). N, protein, $\mathrm{P}$ and $\mathrm{K}$ (in the leaves) of both seasons of each treatment were determined using the methods described by the AOAC (1970) as follows:

The washed and dried materials were ground to fine powder with mortar and pestle and used for dried ashing.

For analysis of $\mathrm{K}$ the powdered plant material $(0.2 \mathrm{~g})$ was taken in precleane and constantly weighed silica crucible and heated in muffle furnace at $400{ }^{\circ} \mathrm{C}$ till there was no evolution of smoke. The crucible was cooled in desicator at room temperature. The ash totally free from carbon moistened with Conc. $\mathrm{H}_{2} \mathrm{SO}_{4}$ and heated on Hot plate till fumes of sulphuric acid get evolved the silica crucible with sulphated ash was again heated at $600{ }^{\circ} \mathrm{C}$ in muffle furnace till weight of sample was constant (3-4 hrs) one gram sulphated ash were taken in beaker which dissolved in $100 \mathrm{ml} 5 \%$ conc. $\mathrm{HCl}$ to obtain solution for determination of $\mathrm{K}$ through flame photometry, standard solution of each mineral was prepared and calibration curve drawn for K element using flame photometry.

For determination of protein and Nitrogen using Micro Kjeldahl method, $1 \mathrm{~g}$ of plant sample taken in a 
Pyrex digestion tube and $30 \mathrm{~mL}$ of conc. $\mathrm{H}_{2} \mathrm{SO}_{4}$ carefully added, then $10 \mathrm{~g}$ potassium sulphate and $14 \mathrm{gm}$ copper sulphate, mixture is placed on sand both on a low flame just to boil the solution, it was further heated till the solution becomes colorless and clear, allowed to cool, diluted with distilled water and transferred $800 \mathrm{~mL}$ Kjeldahl flask, washing the digestion flask, Three or four pieces of granulated zinc and 100 $\mathrm{mL}$ of $40 \%$ caustic soda were added and the flask was connected with the splash heads of the distillation apparatus. Next $25 \mathrm{~mL}$ of $0.1 \mathrm{~N}$ sulphuric acids was taken in the receiving flask and distilled; it was tested for completion of reaction. The flask was removed and titrated against $0.1 \mathrm{~N}$ caustic soda solution using Methyl Red indicator for determination of nitrogen, which in turn give the protein content.

For determination of phosphorous $2 \mathrm{~g}$ sample of plant material taken in $100 \mathrm{ml}$ conical flask two spoons of Darco-G-60 is added followed by $50 \mathrm{~mL}$ of $0.5 \mathrm{M}$ $\mathrm{NaHCo}_{3}$ solution, next flask was corked, and allowed for shaking for $30 \mathrm{~min}$ on shaker. the content was filtered and filtrate was collected in flask from which 5 $\mathrm{ml}$ filtrate was taken in $25 \mathrm{~mL}$ volumetric flask to this 2 drops of 2, 4- paranitrophenol and $5 \mathrm{~N} \mathrm{H}_{2} \mathrm{SO}_{4}$ drop by drop was added with intermittent shaking till yellow color disappear, content was diluted about $20 \mathrm{~mL}$ with distilled water and then $4 \mathrm{~mL}$ ascorbic acid was added then the mixture was shacked well and the intensity of blue color at $660 \mathrm{~nm}$ on colorimeter was measured.
The absorbances were compared and concentrations of phosphorous using standard value were calculated.

\subsection{Statistical analysis}

The average data obtained from both seasons were statistically analyzed using analysis of variance (ANOVA) and the values of least significant difference (LSD) at $1 \%$ and $5 \%$ according to Snedecor and Cochran, 1990).

\section{Results}

\subsection{Effect of NP, trace elements and their interactions on the growth characters}

Plant growth characters such as plant height $(\mathrm{cm})$, leaf number (plant $\left.{ }^{1}\right)$, branch number $\left(\right.$ plant $\left.^{-1}\right)$, umbel number (plant $\left.{ }^{-1}\right)$, herb fresh weight $\left(\mathrm{g} \mathrm{plant}^{-1}\right)$, herb dry weight $\left(\mathrm{g} \mathrm{plant}^{-1}\right)$ and fruit yield $\left(\mathrm{g}\right.$ plant $\left.^{-1}\right)$ in anise, coriander and sweet fennel plants were significantly affected by changes in NP fertilization + trace elements treatments. Thus the various growth characters in general increased under the various NP fertilization levels + trace elements compared with NP fertilization treatments. Highest values of plant growth characters were obtained in the $\mathrm{N} 3 \mathrm{P} 3+$ trace elements treatment. NP and trace elements affected in plant morphology (Table 2). 
Table 2. Effect of NP, trace elements and their interactions on the vegetative growth characters.

\begin{tabular}{|c|c|c|c|c|c|c|c|c|}
\hline \multirow{2}{*}{\multicolumn{2}{|c|}{ Treatments }} & \multicolumn{7}{|c|}{ Vegetative growth characters } \\
\hline & & \multirow{2}{*}{$\begin{array}{c}\begin{array}{c}\text { Plant } \\
\text { height } \\
\text { (cm) }\end{array} \\
\text { sum) }\end{array}$} & \multirow[t]{2}{*}{$\begin{array}{c}\text { Leaf } \\
\text { number } \\
\left(\text { plant }^{-1}\right)\end{array}$} & \multirow[t]{2}{*}{$\begin{array}{l}\text { Branch } \\
\text { number } \\
\left(\text { plant }^{-1}\right)\end{array}$} & \multirow[t]{2}{*}{$\begin{array}{c}\text { Umbel } \\
\text { number } \\
\left(\text { g plant }^{-1}\right)\end{array}$} & \multirow[t]{2}{*}{$\begin{array}{c}\text { Plant fresh } \\
\text { weight } \\
\left(\text { g plant }^{-1}\right)\end{array}$} & \multirow[t]{2}{*}{$\begin{array}{l}\text { plant dry } \\
\text { weight } \\
\left(\text { g plant }^{-1}\right)\end{array}$} & \multirow[t]{2}{*}{$\begin{array}{c}\text { Fruit } \\
\text { yield } \\
\left(\text { g plant }^{-1}\right)\end{array}$} \\
\hline Anise (Pil & pinella & & & & & & & \\
\hline \multirow{4}{*}{$\begin{array}{l}\text { Without } \\
\text { foliar } \\
\text { spray }\end{array}$} & NOP0 & 29.5 & 15.7 & 5.2 & 6.4 & 7.9 & 3.9 & 1.1 \\
\hline & N1P1 & 31.1 & 25.3 & 4.6 & 12.3 & 9.9 & 4.2 & 2.3 \\
\hline & $\mathrm{N} 2 \mathrm{P} 2$ & 40.1 & 27.9 & 7.5 & 19.5 & 14.7 & 9.3 & 5.8 \\
\hline & N3P3 & 45.8 & 31.7 & 8.5 & 21.4 & 22.8 & 10.6 & 5.9 \\
\hline \multicolumn{2}{|c|}{$\begin{array}{l}\text { Over all without } \\
\text { foliar spray }\end{array}$} & 36.9 & 25.2 & 6.5 & 14.9 & 13.8 & 7.0 & 3.8 \\
\hline \multirow{4}{*}{$\begin{array}{l}\text { With } \\
\text { foliar } \\
\text { spray }\end{array}$} & NOP0 & 33.1 & 18.5 & 7.3 & 23.9 & 14.4 & 4.7 & 3.5 \\
\hline & N1P1 & 38.4 & 31.7 & 8.7 & 25.6 & 26.3 & 8.5 & 5.9 \\
\hline & $\mathrm{N} 2 \mathrm{P} 2$ & 44.9 & 37.6 & 9.8 & 31.1 & 33.9 & 10.9 & 7.1 \\
\hline & N3P3 & 53.4 & 45.9 & 10.3 & 33.5 & 36.8 & 11.8 & 7.9 \\
\hline \multicolumn{2}{|c|}{$\begin{array}{l}\text { Over all } \\
\text { foliar spray }\end{array}$} & 42.5 & 33.4 & 9.0 & 28.5 & 27.9 & 9.0 & 6.1 \\
\hline \multirow{4}{*}{$\begin{array}{l}\text { Over all } \\
\text { NP }\end{array}$} & NOP0 & 31.3 & 17.1 & 6.3 & 15.2 & 11.2 & 4.3 & 2.3 \\
\hline & N1P1 & 34.8 & 28.5 & 6.7 & 19.0 & 18.1 & 6.4 & 4.1 \\
\hline & $\mathrm{N} 2 \mathrm{P} 2$ & 42.5 & 32.8 & 8.7 & 25.3 & 24.3 & 10.1 & 6.5 \\
\hline & N3P3 & 49.6 & 38.8 & 9.4 & 27.5 & 29.8 & 11.2 & 6.9 \\
\hline \multicolumn{9}{|c|}{ LSD: 0.05} \\
\hline \multicolumn{2}{|l|}{ NP } & 3.7 & 5.8 & 0.5 & 4.3 & 5.8 & 1.2 & 0.4 \\
\hline \multicolumn{2}{|c|}{ Foliar spray } & 4.9 & 6.3 & 0.9 & 8.9 & 8.9 & 1.4 & 0.6 \\
\hline \multicolumn{2}{|c|}{ NP x foliar spray } & 5.2 & 8.8 & 1.1 & 11.6 & 9.1 & 1.6 & 0.9 \\
\hline \multicolumn{9}{|c|}{$\begin{array}{l}\text { Coriander } \\
\text { (Coriandrum sativum) }\end{array}$} \\
\hline \multirow{4}{*}{$\begin{array}{l}\text { Without } \\
\text { foliar } \\
\text { spray }\end{array}$} & NOP0 & 42.6 & 12.6 & 2,5 & 6.1 & 7.7 & 3.2 & 1.2 \\
\hline & N1P1 & 61.4 & 19.8 & 2.9 & 7.5 & 8.2 & 4.1 & 3.1 \\
\hline & $\mathrm{N} 2 \mathrm{P} 2$ & 69.5 & 39.4 & 3.2 & 11.2 & 10.9 & 5.3 & 5.7 \\
\hline & N3P3 & 70.7 & 43.3 & 4.1 & 22.3 & 16.8 & 7.9 & 6.2 \\
\hline \multicolumn{2}{|c|}{$\begin{array}{l}\text { Over all without } \\
\text { foliar spray }\end{array}$} & 61.1 & 28.8 & 3.4 & 11.8 & 10.9 & 5.1 & 4.1 \\
\hline \multirow{4}{*}{$\begin{array}{l}\text { With } \\
\text { foliar } \\
\text { spray }\end{array}$} & NOP0 & 61.3 & 41.2 & 6.5 & 12.8 & 20.4 & 7.9 & 3.3 \\
\hline & N1P1 & 71.6 & 48.8 & 7.7 & 22.4 & 27.0 & 12.5 & 7.8 \\
\hline & N2P2 & 73.4 & 54.1 & 8.6 & 26.6 & 31.2 & 16.1 & 12.5 \\
\hline & N3P3 & 83.9 & 69.3 & 9.8 & 29.0 & $34 . .0$ & 17.5 & 14.4 \\
\hline
\end{tabular}


Continued...

\begin{tabular}{|c|c|c|c|c|c|c|c|c|}
\hline \multirow{2}{*}{\multicolumn{2}{|c|}{ Treatments }} & \multicolumn{7}{|c|}{ Vegetative growth characters } \\
\hline & & $\begin{array}{c}\text { Plant } \\
\text { height } \\
(\mathrm{cm})\end{array}$ & $\begin{array}{c}\text { Leaf } \\
\text { number } \\
\left(\text { plant }^{-1}\right)\end{array}$ & $\begin{array}{l}\text { Branch } \\
\text { number } \\
\left(\text { plant }^{-1}\right)\end{array}$ & $\begin{array}{c}\text { Umbel } \\
\text { number } \\
\left(\text { g plant }^{-1}\right)\end{array}$ & $\begin{array}{l}\text { Plant fresh } \\
\text { weight } \\
\left(\text { g plant }^{-1}\right)\end{array}$ & $\begin{array}{c}\text { plant dry } \\
\text { weight } \\
\left(\text { g plant }^{-1}\right)\end{array}$ & $\begin{array}{c}\text { Fruit } \\
\text { yield } \\
\left(\text { g plant }^{-1}\right)\end{array}$ \\
\hline \multicolumn{2}{|c|}{$\begin{array}{l}\text { Over all } \\
\text { foliar spray }\end{array}$} & 72.6 & 53.4 & 8.2 & 22.7 & 26.2 & 13.5 & 9.5 \\
\hline \multirow{4}{*}{$\begin{array}{l}\text { Over } \\
\text { all NP }\end{array}$} & N0P0 & 52.0 & 26.9 & 6.5 & 9.5 & 14.1 & 5.6 & 2.3 \\
\hline & N1P1 & 66.5 & 34.3 & 5.3 & 15.0 & 17.6 & 8.3 & 5.5 \\
\hline & $\mathrm{N} 2 \mathrm{P} 2$ & 71.5 & 46.8 & 5.9 & 18.9 & 21.1 & 10.7 & 9.1 \\
\hline & N3P3 & 77.3 & 56.3 & 7.0 & 25.7 & 26.8 & 12.7 & 10.3 \\
\hline \multicolumn{9}{|c|}{ LSD: 0.05} \\
\hline \multicolumn{2}{|l|}{ NP } & 6.8 & 4.9 & 0.3 & 6.5 & 3.4 & 0.9 & 1.1 \\
\hline \multicolumn{2}{|c|}{ Foliar spray } & 7.5 & 8.9 & 1.2 & 7.9 & 5.8 & 1.4 & 2.8 \\
\hline \multicolumn{2}{|c|}{ NP x foliar spray } & 7.9 & 10.6 & 2.3 & 8.4 & 7.5 & 2.5 & 3.3 \\
\hline \multicolumn{9}{|c|}{$\begin{array}{l}\text { Sweet fennel } \\
\text { (Foeniculum vulgare var. 'Dulce') }\end{array}$} \\
\hline \multirow{4}{*}{$\begin{array}{l}\text { Without } \\
\text { foliar } \\
\text { spray }\end{array}$} & NOP0 & 56.3 & 6.8 & 1.4 & 2.3 & 16.2 & 5.2 & 9.7 \\
\hline & N1P1 & 60.6 & 8.8 & 1.9 & 7.6 & 19.4 & 6.7 & 10.6 \\
\hline & $\mathrm{N} 2 \mathrm{P} 2$ & 71.9 & 16.4 & 3.4 & 8.5 & 80.8 & 36.9 & 12.8 \\
\hline & N3P3 & 72.5 & 20.6 & 5.4 & 9.8 & 94.2 & 42.3 & 17.7 \\
\hline \multicolumn{2}{|c|}{$\begin{array}{l}\text { Over all without } \\
\text { foliar spray }\end{array}$} & 65.3 & 13.2 & 3.0 & 7.1 & 52.7 & 22.8 & 12.7 \\
\hline \multirow{4}{*}{$\begin{array}{l}\text { With } \\
\text { foliar } \\
\text { spray }\end{array}$} & NOP0 & 71.8 & 16.7 & 3.4 & 11.0 & 137.6 & 34.1 & 18.9 \\
\hline & N1P1 & 72.5 & 22.9 & 4.4 & 13.7 & 233.6 & 52.9 & 21.3 \\
\hline & $\mathrm{N} 2 \mathrm{P} 2$ & 83.9 & 25.8 & 5.6 & 19.8 & 235.9 & 89.6 & 22.8 \\
\hline & N3P3 & 89.9 & 32.6 & 7.8 & 22.9 & 257.8 & 99.1 & 27.8 \\
\hline \multicolumn{2}{|c|}{$\begin{array}{l}\text { Over all foliar } \\
\text { spray }\end{array}$} & 79.5 & 24.5 & 5.3 & 16.9 & 216.3 & 67.0 & 22.7 \\
\hline \multirow{4}{*}{$\begin{array}{l}\text { Over all } \\
\text { NP }\end{array}$} & NOP0 & 64.1 & 11.8 & 2.4 & 6.7 & 76.9 & 19.7 & 14.3 \\
\hline & N1P1 & 66.6 & 15.9 & 3.2 & 10.7 & 126.5 & 29.8 & 16.0 \\
\hline & $\mathrm{N} 2 \mathrm{P} 2$ & 77.9 & 21.1 & 4.5 & 14.2 & 158.4 & 63.3 & 17.8 \\
\hline & N3P3 & 81.2 & 26.6 & 6.6 & 16.4 & 176.0 & 70.7 & 22.8 \\
\hline \multicolumn{9}{|c|}{ LSD: 0.05} \\
\hline \multicolumn{2}{|l|}{ NP } & 6.8 & 5.4 & 1.0 & 3.6 & 13.3 & 12.4 & 3.6 \\
\hline \multicolumn{2}{|c|}{ Foliar spray } & 7.1 & 7.7 & 1.1 & 4.9 & 23.7 & 23.8 & 4.8 \\
\hline \multicolumn{2}{|c|}{ NP x foliar spray } & 8.2 & 8.8 & 1.7 & 5.3 & 31.8 & 25.8 & 5.7 \\
\hline
\end{tabular}


3.2 Effect of NP, trace elements and their interactions on the essential oil content

As shown in Table 3 essential oil content increased at all NP treatments, trace elements and NP + trace elements levels. The highest accumulation of essential oil was recorded at the highest NP level (N3P3) $\mathrm{x}$ trace elements interaction compared with control treatment (NOP0).

Table 3. Effect of NP, trace elements and their interactions on the chemical content.

\begin{tabular}{|c|c|c|c|c|c|c|c|c|c|}
\hline \multirow{2}{*}{ Treatments } & & \multicolumn{8}{|c|}{ Chemical content (\%) } \\
\hline & & $\begin{array}{l}\text { Essen- } \\
\text { tial oil }\end{array}$ & $\begin{array}{c}\text { Fixed } \\
\text { oil }\end{array}$ & $\begin{array}{c}\text { Total } \\
\text { carbohydrates }\end{array}$ & $\begin{array}{c}\text { Soluble } \\
\text { sugars }\end{array}$ & $\begin{array}{c}\text { Crude } \\
\text { protein }\end{array}$ & $\mathbf{N}$ & $\mathbf{P}$ & $\mathbf{K}$ \\
\hline \multicolumn{10}{|c|}{ Anise (Pimpinella anisum) } \\
\hline \multirow{4}{*}{$\begin{array}{l}\text { Without foliar } \\
\text { spray }\end{array}$} & NOP0 & 2.4 & 4.3 & 6.9 & 2.1 & 10.6 & 1.7 & 0.5 & 1.3 \\
\hline & N1P1 & 2.5 & 5.6 & 8.9 & 2.9 & 11.3 & 1.8 & 0.7 & 1.4 \\
\hline & $\mathrm{N} 2 \mathrm{P} 2$ & 2.8 & 6.8 & 10.6 & 3.1 & 11.9 & 1.9 & 0.9 & 1.5 \\
\hline & N3P3 & 2.9 & 6.9 & 12.8 & 3.1 & 13.1 & 2.1 & 0.9 & 1.5 \\
\hline \multicolumn{2}{|c|}{$\begin{array}{l}\text { Over all without } \\
\text { foliar spray }\end{array}$} & 2.7 & 5.9 & 9.8 & 2.8 & 11.7 & 1.9 & 0.8 & 1.4 \\
\hline \multirow{4}{*}{$\begin{array}{l}\text { With } \\
\text { foliar spray }\end{array}$} & NOP0 & 2.5 & 5.6 & 8.9 & 2.8 & 11.9 & 1.9 & 1.2 & 1.7 \\
\hline & $\mathrm{N} 1 \mathrm{P} 1$ & 2.7 & 7.5 & 9.8 & 3.5 & 13.8 & 2.2 & 1.4 & 1.9 \\
\hline & $\mathrm{N} 2 \mathrm{P} 2$ & 2.9 & 8.9 & 13.6 & 3.9 & 14.4 & 2.3 & 1.9 & 2.1 \\
\hline & N3P3 & 3.3 & 9.7 & 15.9 & 4.5 & 15.6 & 2.5 & 2.0 & 2.6 \\
\hline \multicolumn{2}{|c|}{ Over all trace elements } & 2.9 & 7.9 & 12.1 & 3.7 & 14.0 & 2.2 & 1.6 & 2.1 \\
\hline \multirow{4}{*}{ Over all NP } & NOP0 & 2.5 & 5.0 & 7.9 & 2.5 & 11.3 & 1.8 & 0.9 & 1.5 \\
\hline & N1P1 & 2.6 & 6.6 & 9.4 & 3.2 & 12.6 & 2.0 & 1.1 & 1.6 \\
\hline & $\mathrm{N} 2 \mathrm{P} 2$ & 2.9 & 7.9 & 12.1 & 3.5 & 13.2 & 2.1 & 1.4 & 1.8 \\
\hline & N3P3 & 3.1 & 8.3 & 14.4 & 3.8 & 14.4 & 2.3 & 1.5 & 2.1 \\
\hline \multicolumn{10}{|l|}{ LSD: 0.05} \\
\hline \multicolumn{2}{|l|}{ NP } & 0.1 & 1.1 & 1.3 & 0.4 & 1.3 & 0.1 & 0.1 & 0.1 \\
\hline \multicolumn{2}{|c|}{ Trace elements } & 0.2 & 2.3 & 1.9 & 0.8 & 2.1 & 0.2 & 0.2 & 0.1 \\
\hline \multicolumn{2}{|c|}{ NP $x$ Trace elements } & 0.3 & 3.1 & 3.1 & 1.2 & 2.5 & 0.2 & 0.2 & 0.2 \\
\hline \multicolumn{10}{|c|}{ Coriander (Coriandrum sativum) } \\
\hline \multirow{4}{*}{$\begin{array}{l}\text { Without } \\
\text { foliar spray }\end{array}$} & NOP0 & 0.2 & 2.5 & 5.8 & 1.6 & 10.0 & 1.4 & 0.3 & 2.3 \\
\hline & N1P1 & 0.3 & 3.6 & 6.8 & 1.9 & 11.9 & 1.7 & 0.4 & 2.5 \\
\hline & $\mathrm{N} 2 \mathrm{P} 2$ & 0.3 & 5.8 & 9.8 & 2.0 & 12.5 & 1.9 & 0.4 & 2.6 \\
\hline & N3P3 & 0.3 & 6.8 & 9.9 & 2.2 & 13.8 & 2.1 & 0.5 & 2.9 \\
\hline \multicolumn{2}{|c|}{$\begin{array}{l}\text { Over all without } \\
\text { foliar spray }\end{array}$} & 0.3 & 4.7 & 8.1 & 1.9 & 12.1 & 1.8 & 0.4 & 2.6 \\
\hline
\end{tabular}


Continued...

\begin{tabular}{|c|c|c|c|c|c|c|c|c|c|}
\hline \multirow{2}{*}{ Treatments } & & \multicolumn{8}{|c|}{ Chemical content $(\%)$} \\
\hline & & $\begin{array}{l}\text { Essen- } \\
\text { tial oil }\end{array}$ & $\begin{array}{c}\text { Fixed } \\
\text { oil }\end{array}$ & $\begin{array}{c}\text { Total } \\
\text { carbohydrates }\end{array}$ & $\begin{array}{c}\text { Soluble } \\
\text { sugars }\end{array}$ & $\begin{array}{c}\text { Crude } \\
\text { protein }\end{array}$ & $\mathbf{N}$ & $\mathbf{P}$ & $\mathbf{K}$ \\
\hline \multirow{4}{*}{$\begin{array}{l}\text { With } \\
\text { Foliar spray }\end{array}$} & NOP0 & 0.3 & 4.9 & 7.9 & 3.2 & 20.0 & 1.8 & 0.4 & 2.4 \\
\hline & N1P1 & 0.4 & 5.9 & 8.9 & 3.4 & 21.3 & 1.9 & 0.5 & 2.9 \\
\hline & $\mathrm{N} 2 \mathrm{P} 2$ & 0.5 & 8.7 & 12.9 & 3.9 & 24.4 & 2.3 & 0.8 & 3.3 \\
\hline & N3P3 & 0.5 & 9.6 & 13.7 & 4.4 & 27.5 & 4.4 & 0.9 & 3.5 \\
\hline \multicolumn{2}{|c|}{ Over all foliar spray } & 0.4 & 7.3 & 10.9 & 3.7 & 23.3 & 2.6 & 0.7 & 3.0 \\
\hline \multirow{4}{*}{ Over all NP } & N0P0 & 0.3 & 3.7 & 6.9 & 2.4 & 15.0 & 1.6 & 0.4 & 2.4 \\
\hline & N1P1 & 0.4 & 4.8 & 7.9 & 2.6 & 16.6 & 1.8 & 0.5 & 2.7 \\
\hline & $\mathrm{N} 2 \mathrm{P} 2$ & 0.4 & 7.3 & 11.4 & 3.0 & 18.5 & 2.1 & 0.6 & 3.0 \\
\hline & N3P3 & 0.4 & 8.2 & 11.8 & 3.3 & 20.7 & 3.3 & 0.7 & 3.2 \\
\hline \multicolumn{10}{|l|}{ LSD: 0.05} \\
\hline \multicolumn{2}{|l|}{ NP } & NS & 2.1 & 2.1 & 0.2 & 1.6 & 0.2 & 0.1 & 0.3 \\
\hline \multicolumn{2}{|l|}{ Foliar spray } & NS & 2.1 & 2.3 & 0.3 & 1.7 & 0.3 & 0.2 & 0.3 \\
\hline \multicolumn{2}{|c|}{ NP $x$ foliar spray } & NS & 2.2 & 4.6 & 0.9 & 2.0 & 0.4 & 0.3 & 0.4 \\
\hline \multicolumn{10}{|c|}{ Sweet fennel (Foeniculum vulgare var. 'Dulce') } \\
\hline \multirow{4}{*}{$\begin{array}{l}\text { Without foliar } \\
\text { spray }\end{array}$} & NOP0 & 1.3 & 1.2 & 8.6 & 1.7 & 7.5 & 1.2 & 0.3 & 2.1 \\
\hline & N1P1 & 1.4 & 1.9 & 9.8 & 1.9 & 10 & 1.6 & 0.4 & 2.3 \\
\hline & $\mathrm{N} 2 \mathrm{P} 2$ & 1.5 & 2.2 & 10.9 & 2.1 & 11.9 & 1.9 & 0.4 & 2.4 \\
\hline & N3P3 & 1.5 & 2.9 & 11.1 & 2.2 & 13.1 & 2.1 & 0.5 & 2.6 \\
\hline \multicolumn{2}{|c|}{$\begin{array}{l}\text { Over all without } \\
\text { foliar spray }\end{array}$} & 1.4 & 2.1 & 10.1 & 2.0 & 10.6 & 1.7 & 0.4 & 2.4 \\
\hline \multirow{4}{*}{$\begin{array}{l}\text { With } \\
\text { foliar spray }\end{array}$} & N0P0 & 1.5 & 2.3 & 9.6 & 2.0 & 11.9 & 1.9 & 0.5 & 2.8 \\
\hline & N1P1 & 1.7 & 3.6 & 12.7 & 2.3 & 15.6 & 2.5 & 0.6 & 2.9 \\
\hline & $\mathrm{N} 2 \mathrm{P} 2$ & 1.8 & 4.8 & 14.8 & 2.8 & 18.1 & 2.9 & 0.8 & 3.6 \\
\hline & N3P3 & 2.2 & 4.9 & 16,8 & 3.3 & 21.9 & 3.5 & 0.7 & 3.8 \\
\hline \multicolumn{2}{|c|}{ Over all foliar spray } & 1.8 & 3.9 & 12.4 & 2.6 & 16.9 & 2.7 & 0.7 & 3.3 \\
\hline \multirow{4}{*}{ Over all NP } & N0P0 & 1.4 & 1.8 & 9.1 & 1.9 & 9.7 & 1.6 & 0.4 & 2.5 \\
\hline & N1P1 & 1.6 & 2.8 & 11.3 & 2.1 & 12.8 & 2.1 & 0.5 & 2.6 \\
\hline & $\mathrm{N} 2 \mathrm{P} 2$ & 1.7 & 3.5 & 12.9 & 2.5 & 15.0 & 2.4 & 0.6 & 3.0 \\
\hline & N3P3 & 1.9 & 3.9 & 11.1 & 2.8 & 17.5 & 2.8 & 0.6 & 3.2 \\
\hline \multicolumn{10}{|l|}{ LSD: 0.05} \\
\hline \multicolumn{2}{|l|}{ NP } & 0.2 & 0.5 & 1.1 & 0.1 & 2.4 & 0.3 & NS & 0.1 \\
\hline \multicolumn{2}{|l|}{ Foliar spray } & 0.3 & 1.1 & 1.4 & 0.3 & 3.0 & 0.5 & NS & 0.1 \\
\hline \multicolumn{2}{|c|}{ NP $\mathrm{x}$ foliar spray } & 0.3 & 2.4 & 1.8 & 0.5 & 3.9 & 0.6 & NS & 0.2 \\
\hline
\end{tabular}




\subsection{Effect of NP, trace elements and their interactions on the fixed oil content}

Fixed oil content increased with NP, trace elements and their interactions (Table 3). However, the highest fixed oil content resulted from N3P3 + trace elements treatment compared with control.

\subsection{Effect of NP, trace elements and their interactions on the Total carbohydrates and soluble sugars content}

Total carbohydrates and soluble sugars content increased with NP fertilization, trace elements and the NP fertilization $\times$ trace elements interaction (Table 3). However, the highest total soluble sugars content resulted from N3P3 + trace elements treatment compared with control.

\subsection{Effect of NP, trace elements and their interactions on the crude protein content}

The accumulation of protein in anise, coriander and sweet fennel plants leaves was promoted by applying various levels of NP, trace elements and their interactions (Table 3). The highest protein content resulted from N3P3 + trace elements treatment.

\subsection{Effect of NP, trace elements and their interactions on mineral content}

Addition of NP ameliorated the increase in NPK contents (\%) with increasing NP fertilization. Control (NOP0) treatment resulted in the lowest nutrient accumulation while the highest mineral content was observed in the $\mathrm{N} 3 \mathrm{P} 3+$ trace elements treatment.

\section{Discussion}

The positive effects of these treatments (NP, trace elements and their interactions) may be due to the important physiological role of $\mathrm{N}$; $\mathrm{N}$ plays an important role in synthesis of the plant constituents through the action of different enzymes activity and protein synthesis (Jones et al., 1991) that reflected on an increase in growth parameters and chemical constituents of anise, coriander and sweet fennel plants. The obtained results are in accordance with those obtained by previous literature. $\mathrm{N}$ is a necessary component of several vitamins. $\mathrm{N}$ improves the quality and quantity of dry matter in leafy plants and protein in grain crops (Silva and Uchida, 2000). Increase the $\mathrm{N}$ fertilizer caused a significant increase in the seed yield of Japanese mint (Mentha arvensis L) (Randhawa et al., 1996; Munsi, 1992). N fertilization increased the vegetative growth, essential oil, fixed oil, total carbohydrates, soluble sugars and NPK content of Nigella sativa L. plants (Khalid, 2001). Zheljazkov and Margina (1996) established that vegetative growth and essential oil (yield and constituents) of Mentha piperita and Mentha arvensis were increased as $\mathrm{N}$ fertilizer increase (Saxena and Singh, 1998). Arabaci and Bayram (2004) found that $\mathrm{N}$ fertilizer increased the amount of green herb yield, drug herb yield, drug leaves, essential oil (\% \& yield) of basil (Ocimum basilicum L.). $\mathrm{N}$ fertilization increased the dry weight of Mentha. x piperita, linalool chemotype (Luciana et al., 2010). P leads to enhanced herb and essential oil yields of different mint species (Kothari et al., 1987). The $\mathrm{P}$ had a stimulating effect on the growth parameters, total carbohydrates, soluble sugars, mineral contents and on the percentage of essential oil production from chamomile flow- 
ers compared with the control (Nassar et al., 2004). Protein results be due to $\mathrm{N}$ which has an influence on the ribosome structure and the biosynthesis of some hormones (gibberellines, auxins and cytokinins) involved in protein synthesis (Jones et al., 1991); P activates coenzymes for amino acid production used in protein synthesis (Espinosa et al., 1999); Trace elements are redox-active that makes them essential as catalytically active cofactors in enzymes, others have enzyme-activating functions, and yet others fulfill a structural role in stabilizing proteins (Hänsch and Mendel, 2009). Hussien (1995) reported that NP fertilization was more affect on the dill essential oil. It was established that plant height, branching and essential oil content were increased with increasing NP fertilizer rates. However, plant leaves were not significantly affected by the increase of NP rates (Zheljazkov and Margina, 1996). Fresh material and essential oil yields of peppermint (Mentha X piperita L.) were increased by the increase in NP levels (Jeliazkova, et al., 1999). NP treatments produced the highest growth and essential oil of garden thyme (Thymus vulgaris L.) compared with the control treatment (Sharafzadeh, 2011). Spraying of trace elements under sandy soil conditions resulted in a significant increase in vegetative characters, essential oil, NPK and total carbohydrate content of Trachyspermum ammi L (Abd El- Wahab and Mohamed, 2008). Nasiri et al. (2010) showed that flower yield, essential oil (\% and yield) increased by foliar application of micronutrients compared with control untreated. Essential oil, growth and yield of onion plants significantly increased by the application of micronutrients compared with control plants (El-Tohamy et al., 2009). NP fertilization + trace elements increased the vegetative growth, essential oil, fixed oil, total carbohydrates, soluble sugars, protein N, P and K of some medicinal Apiaceae plants (Khalid, 1996). Increasing the essential minerals according to the NP, trace elements and their interac- tions treatments may be due to the increase in the dry matter of plant materials (Kandeel 1991; Khalid 1996; Silva and Uchida, 2000; Mahmoud, 2002). The effect of NP, trace elements and their interactions treatments on essential oil may be due to its effect on enzyme activity and metabolism of essential oil production in peppermint plant (Burbott and Loomis, 1969). The results of fixed oil agree with those obtained by Khalid, 2001 on Nigella sativa L. plant; Espinosa et al. (1999) who indicated that NP and trace elements plays an important role in various metabolism processes such as fatty acid (fixed oil) synthesis.

\section{Conclusions}

It may be concluded that NP + trace elements had a significant effect on anise, coriander and sweet fennel plants which positively affect on growth and chemical constituent's of these three plants grow under arid regions in Egypt.

\section{References}

Adebooye, O.C., Oloyede, F.M. 2007. Effect of phosphorus on the fruit yield and food value of two landraces of Trichosanthes cucumerina L. Cucurbitaceae. Food Chem. 100, 1259-1264.

Abd-Allah, A.M., Adam, S.M., Abou- Hadid, A.F. 2001. Productivity of green cowpea in sandy soil as influenced by different organic manure rates and sources. Egypt. J. Hort. Sci. 28 ,3, 331-340.

Abd El- Wahab, A., Mohamed, A. 2008. Effect of some trace elements on growth, yield and chemical constituents of Trachyspermum ammi L (Ajowan) plants under sinai conditions. Res. J. Agric. Bio. Sci. 4 ,6, 717-724.

Akbarinia, A., Jahanfar, D., Beygifarzad, M. 2007. Effect of nitrogen fertilizer and plant density on seed yield, essential oil and fixed oil content of 
Coriandrum sativum L. Int. J. Med. Arom. Plants. $22,34,410-419$.

Arabaci, O., Bayram, E. 2004. The effect of nitrogen fertilization and plant densities of some agronomic and technologic characteristics of Ocimum basilicum L. (Basil). J. Agron. 3 ,4, 255-262.

Arnon, D.I., Hoagland, D.R. 1943. Composition of the tomato plant as influenced by nutrient supply, in relation to fruiting. Bot. Gaz. 104, $576-592$.

Ashraf, M., Ali, Q., Iqba, Z. 2006. Effect of nitrogen application rate on the content and composition of oil, essential oil and minerals in black cumin (Nigella sativa L.) seeds. J. Sci. Food Agric. 8630,60, 871-876.

Association of Official Agricultural Chemistry (AOAC) 1970 Official Methods Analysis $10^{\text {th }}$ Washington, D. C.

Baranauskienne, R., Venskutonis, P.R., Viskelis, P., Dambrausiene, E. 2003. Influence of nitrogen fertilizer on the yield and composition of thyme (Thymus vulgaris). J. Agric. Food Chem. 51, 7751-7758.

Besmer, Y.L., Koide, R.T. 1999. Effect of mycorrhizal colonization and phosphorus on ethylene production by snapdragon (Antirrhinum majus L.) flowers. Mycorrh. 9, $161-166$.

Burbott, A.J., Loomis, D. 1969. Evidence for metabolic turnover monoterpene in peppermint. Plant Phys. 44, 173-179.

Burt, S., 2004. Essential oils: their antibacterial properties and potential applications in foods-a review. Int. J. Food Microbiol. 94, 223-253.

Chithra, V., Leelamma, S. 2000. Coriandrum sativum effect on lipid metabolism in 1, 2-dimethyl hydrazine induced colon cancer. J. Ethnopharm. 71, 457-463.
Ciha, A.J., Brun, W.A. 1978. Effect of pod removal on nonstructural carbohydrate concentration in soybean tissue. Crop Sci. 18, 773-776.

Clevenger, J.F. 1928. Apparatus for determination of essential oil. J. Amer. Pharm. Assoc. 17, 346-349.

Cottenie, A., Verloo, M., Kiekens, L., Velghe, G., Camerlynck, R. 1982. Chemical analysis of plant and soil. Laboratory of Analytical and Agrochemistry, State Univ., Ghent, Belgium, PP. 100 - 129.

Czygan, F., Anis, C. 1992. (Anisi fructus DAB 10) - Pimpinella anisum. Z. Phytother. 13, 101-106.

Dubois, M., Gilles, K.A., Hamilton, J.K., Roberts, P.A., Smith, F. 1956. Phenol sulphuric acid method for carbohydrate determination. Annal. Chem. 28, 350-359.

Jackson, M.L. 1973. Soil chemical analysis. Published by prentice Hall Indian Private Limited. M.97, Connght Citrus, New Delhi-1. PP. 200 - 250.

Economakis, C., Skaltsa, H., Demetzos, C., Soković, M.C.A. 2002. Effect of phosphorus concentration of the nutrient solution on the volatile constituents of leaves and bracts of Origanum dictamnus. $J$. Agric. Food Chem. 50,22, 6276-6280.

Egle, K., Manske, G., Römer, W., Vlek, P.L.G. 1999. Improved phosphorus efficiency of three new wheat genotypes from CIMMYT in comparison with an older Mexican variety. J. Plant Nut Soil Sci. $162,353-358$.

El-Tohamy, W.A., Khalid, A. K., El-Abagy, H.M., Abou-Hussein, S.D. 2009. Essential oil, growth and yield of onion (Allium Cepa L.) in response to foliar application of some micronutrients. Aus. J. Bas. App. Sci. 3,1, 201-205.

Emamghoreishi, M., Khasaki, M., Aazam, M.F. 2005. Coriandrum sativum: evaluation of its anxiolytic effect in the elevated plus-maze. J. Ethnopharm. 96, 365-370. 
Espinosa, M., Turner, B.L., Haygarth, P.M. 1999. Preconcentration and separation of trace phosphorus compounds in soil leachate. J. Environ. Qual. 29, $1497-1504$

Eyres, G., Dufour, J.P., Hallifax, G., Sotheeswaran, S., Marriott, P. J. 2005. Identification of characterimpact odorants in coriander and wild coriander leaves using gas chromatography-olfactometry (GCO) and comprehensive two-dimensional gas chromatography-time of light mass spectrometry (GC-TOFMS). J. Sep. Sci. 28, 1061 - 1074.

Ezz El-Din, A.A., Aziz, E.E., Hendawy, S.F., Omer, E.A. 2010. Impact of phosphorus nutrition and number of cutting on growth, yield and active constituents of artichoke. Int. J. Acad. Rese. 2,4 , 240- 244.

Feller, I.C. 1995. Effects of nutrient enrichment on growth and herbivory of Dwarf Red Mangrove (Rhizophora Mangle). Ecol. Monog. 65, 477-505.

Hänsch, R., Mendel, R.R. 2009. Physiological functions of mineral micronutrients $(\mathrm{Cu}, \mathrm{Zn}, \mathrm{Mn}, \mathrm{Fe}$, $\mathrm{Ni}, \mathrm{Mo}, \mathrm{B}, \mathrm{Cl})$. Current opinion in plant biology. $12,3,259-266$.

Hänsel, R., Sticher, O., Steinegger, E. 1999. Pharmakognosie-Phytopharmazie, 6th ed., Springer-Verlag, Berlin, pp. 692-695.

Haupt, C. 1986. New vegetables for greenhouse cultivation in winter. Celery and florence fennel. Gart. Tid. 107, $723-725$.

Hellal, F.A., Mahfouz S.A., Hassan, F.A.S. 2011. Partial substitution of mineral nitrogen fertilizer by bio-fertilizer on (Anethum graveolens L.) plant. Agri. Biol. J. North Amer. 4, 652 - 660.

Hornok, L. 1980. Effect of nutrition supply on yield of dill (Anithum graveolens L.) and the essential oil content. Acta Hort. 96, 337-342.

Hussien, M.S. 1995. Response of growth, yield and essential oil of coriander and dill to different nitrogen sources. Egyp. J. Hort. Sci. 22 , 1, 1-10.
Jones, I.B., Wolf, B., Milles, H.A. 1991. Plant analysis handbook. Macro- Micro Publishing. Inc., pp. 213.

Jeliazkova, E.A., Zheljazkov, V.D., Craker, L.E., Yankov, B., Georgieva, T. 1999. NPK fertilizer and yields of peppermint, Mentha X piperita. Acta Hort. 502, 231-236.

Kamel, M., Karim Hosni, M.B.T., Thouraya, C., Mohamed, E.K., Cserni, I. 1994. The effect of nutrients and variety on keeping quality during storage of fennel (Foeniculum vulgare Mill. subsp. Capillaceaum Gilib. var. Azoricum). Acta Hort. 468, $185-189$.

Kandeel, A., 1991. Influence of soil and foliar nutrition on growth and volatile oil content of parsley (Petroselinum crispum Mill). Ann. Agric. Sci. 36,1, 155-162.

Karthikeyan, B., Abdul - Jaleel, C., Changxing, Z., Joe, M.M., Srimannarayan, J., Deiveekasundaram, M. 2008. The effect of AM fungi and phosphorous level on the biomass yield and ajmalicine production in Catharanthus roseus. EurAsian J. Bio. Sci. 2, 26-33.

Khalid, K.A. 1996. Effect of fertilization on the growth, yield and chemical composition of some medicinal umbelleferous plant. MSc. thesis, Fac. Agric., Al-Azhar Univ., Cairo, Egypt, pp1-5.

Khalid, K.A. 2001. Physiological studies on the growth and chemical composition of Nigella sati$v a$ L. plants. Ph.D. thesis, Fac. Agric., Ain- Shams Univ., Cairo, Egypt.

Kothari, S.K., Singh, V., Singh, K. 1987. Effect of rates and methods of phosphorus application on herb and oil yields and nutrient concentrations in Japanese mint (Mentha arvensis L.). J. Agric Sci. $108,691-693$.

Lawless, J. 1999. The Illustrated Encyclopedia of Essential Oils, The Bridgewater Book Company Ltd., Shaftesbury, pp. 44-45. 
Letchamo, W. 1986. Effect of the trace elements manganese and molybdenum on the leaf yield and content of cardiac glucodides in foxglove, Digitalis grandiffora, Mill. Acta Hort. 188, 215 - 220.

Luciana, W.P., Castro, A., Deschamps, C., Biasi, L.A., Scheer, A.P., Bona, C. 2010. Development and essential oil yield and composition of mint chemotypes under nitrogen fertilization and radiation levels. World Congress of Soil Science, Soil Solutions for a Changing World $1-6$ August 2010, Brisbane, Australia.

Ma, Q., Longnecker, N., Atkins, C. 2001. Varying phosphorus supplies and development, growth and seed yield in narrowleafed lupin. Plant and Soil. 239, $79-85$.

Mahmoud, S.M. 2002. Effect of water stress and NPK fertilisation on growth and resin content of Grindella camporum greene. Acta Hort. 576, 289-294.

Marotti, M., Dellacecca, V., Piccaglia, R., Giovanelli, E., Palevitch, D., Simon, J.E. 1993. Agronomic and chemical evaluation of three "varieties" of Foeniculum vulgare Mill. Acta Hort. 331, 63 - 69.

Munsi, P.S. 1992. Nitrogen and phosphors nutrition response in Japanese mint cultivation. Acta Hort. 306, 436 - 443.

Nasiri, Y., Zehtab-Salmasi, S., Nasrullahzadeh, S., Najafi, N., Ghassemi-Golezani, K. 2010. Effects of foliar application of micronutrients (Fe and $\mathrm{Zn}$ ) on flower yield and essential oil of chamomile (Matricaria chamomilla L.). J. Med. Plants Res. 4, 17, 1733-1737.

Nassar, A.H., Hashim, M.F., Hassan, N.S., Abo-zaid, H. 2004 Effect of gamma irradiation and phosphorus on growth and oil production of chamomile (Chamomilla recutita L. Rauschert). Int. J. Agric. Biol. 6,5, 776 - 780.

Nikolova, A., Kozhuharova, K., Zheljazkov,V.D., Craker. L. E. 1999. Mineral nutrition of chamo- mile (Chamomilla recutta (L.). Acta Hort. 502, $203-208$.

Papadopoulos, A.P. 1994. Growing greenhouse seedless cucumbers in soil and soilless media. Agriculture and Agri-Food Canada, Ottawa, ON.

Rao, B.R. ; Simgh, K., Kaul, P.N. ;Bhattacharya, A.K., 1989. The effect of plant spacings and application of $\mathrm{N}$ and $\mathrm{P}$ fertilizers on the productivity and nutrient uptake of davana (Artemisia pallens Wall.). International journal of tropical agriculture. 7,3-4, 229-236.

Rajeswara, R.B.R., Singh, K., Kaul, P.N., Bhattacharya, A.K. 1989. The effect of plant spacings and application of $\mathrm{N}$ and $\mathrm{P}$ fertilizers on the productivity and nutrient uptake of davana (Artemisia pallens Wall.) Int. J. Trop. Agric. 7,3-4, 229-236.

Randhawa, G.S., Gill, B.S., Saini, S.S., Singh, J. 1996. Effect of plant spacing and nitrogen levels on the seed yield of dill seed (Anethum graveolens L.). Acta Hort. 426, 623 - 628.

Robert, M.D. 1986. Plant physiology. Robert Johnson, Publisher (PWS), A division of Wadsworth Inc., Boston, USA, 786 pp.

Saxena, A., Singh, J.N. 1998. Effect of irrigation, mulch and nitrogen on yield and composition of Japanese mint (Mentha arvensis) oil. Ind. J. Agron. 43, $179-182$.

Senthil Kumar, T., Swaminathan, V., Kumar, S. 2009. Influence of nitrogen, phosphorus and biofertilizers on growth, yield and essential oil constituents in Raton crop of davana (Artemisia pallens Wall.). Elec. J. Environ. Agric. Food Chem. 8 ,2, 86-95.

Shamsi, S.R.A.,Whitehead, F.H. 1977. Comparative ecophysiology of Epilobium hirsutum L. and Lythrum salicaria L. III Mineral nutrition. J. Ecol. $65,55-70$.

Sharafzadeh, S. 2011. Effect of nitrogen, phosphorous and potassium on growth, essential oil and total 
phenolic content of garden thyme (Thymus vulgaris L.). Advanc. Environ. Biol. 5,4, 699 - 703.

Silva J. A., Uchida, R. 2000. Plant Nutrient Management in Hawaii's Soils, Approaches for Tropical and Subtropical Agriculture, University of Hawaii at Manoa.

Snedecor, G.W., Cochran, W.G. 1990. Statistical Methods. $11^{\text {th }}$ Ed. Iowa State Univ., Press. Ames, Iowa, USA.

Steinegger, E., Hansel, R. 1988. Lehrbuch der Pharmakognosie und Phytopharmazie. Berlin: Springer-Verlag, p. 278.

Stewart, C.L.S., Lovett-Doust, L. 2003. Effect of phosphorus treatment on growth and yield in the medicinal herb Calendula officinalis L. (Standard
Pacific) under hydroponic cultivation. Can. J. Plant Sci. 4, $611-617$.

Stuart, M. 1982. Herbs and Herbalism. Van Nostrand Reinhold, New York, pp. 62 - 64.

Tyler, V.E. Brady, L.R., Robbers, J.E. 1988. Pharmacognosy, 9th ed., Lea and Fabiger, Philadelphia, p. 125.

Wangensteen, H., Samuelsen, A.B., Malterud, K.E. 2004. Antioxidant activity in extracts from coriander. Food Chem. 88, 293 - 297.

Zheljazkov, V., Margina, A. 1996. Effect of nitrogen doses of fertilizer application on quantitative and qualitqtive characters of mint. Acta Hort. 426, 579-592 